\title{
Preludes to the Lattice Compatibility Theory LCT: Urbach Tailing Controversial Behavior in Some Nanocompounds
}

\author{
K. Boubaker \\ École Supérieure de Sciences et Techniques de Tunis (ESSTT), Université de Tunis, 63 Rue Sidi Jabeur, Mahdia 5100, Tunisia \\ Correspondence should be addressed to K. Boubaker, mmbb11112000@yahoo.fr
}

Received 19 July 2012; Accepted 6 September 2012

Academic Editors: A. M. Ali, A. Longo, and R. Martinez-Sanchez

Copyright $\odot 2012$ K. Boubaker. This is an open access article distributed under the Creative Commons Attribution License, which permits unrestricted use, distribution, and reproduction in any medium, provided the original work is properly cited.

We try to give an explanation to the unexpected Urbach tailing associated to some phenomena which occur in some nano-compounds lattices. The provided discussion and notes are detailed here as a prelude to the lattice compatibility theory (LCT), a guide to a plausible understanding of this unsolved intrigue.

\section{Introduction}

Urbach tailing alterations inside nanostructured lattices (Figure 1) have been always attributed to atomic-scale topological disorder, unexpected hybrid excitonic transitions, or topological filament occurrence [1-4]. In the beginning of the last decade, Urbach tailing has been attributed to the hydrogen content, and precisely the different $\mathrm{E}_{04}$ and $\mathrm{sp}^{2}$ contents inside nanofilms [5]. Another more realistic explanation has been provided earlier by Dow and Redfield [6]. This explanation claims that Urbach tailing is due to the broadening of the exciton absorption band which occurs when charged impurities in the lattice induce phononinduced microelectric fields. This phenomenon has been also described, in II-VI compound semiconductors, as a reduction of holes-electrons Coulombic interactions which results in more free excitons, as long as exciton radii are larger than those of alkali halides. Many other causes have been proposed in the relevant literature [7-10].

In this paper, a prelude to the micro-chemistry-related Lattice Compatibility Theory (LCT) is provided as an attempt to explain Urbach tailing alterations in nano-structured lattices. The study is organized as follows. In Section 2, the experimental details and motivations are presented along with the studied nanomaterials patterns. In Section 3, the fundaments of the proposed Lattice Compatibility Theory LCT are discussed. Last section is a conclusion which summarizes the aim methods and perspectives of the work.

\section{Experimental Details and Motivations}

In this study, targeted nano-materials are some bismuth oxides BSO, BGO, and BTO. These nano-materials are technologically interesting material, because they can function as a Pockels readout optical modulation (PROM) device and photorefractive sensors [11-18]. Crystals of these oxides were grown from highly purified $\mathrm{Bi}_{2} \mathrm{O}_{3}, \mathrm{SiO}_{2}$ and presintered $\mathrm{BGO}$, and BTO powders using the Czochralski technique [19]. Elaboration was carried out in room atmosphere and the growing temperature was about $1110^{\circ} \mathrm{C}$. Doped nanocrystals were prepared with doping content of $0.02 \mathrm{~mol} \%$ in the starting melt and the samples were reexcited each optically by UV radiations for photochromic effect studies.

In order to understand Urbach tailing alteration following doping agent insertion in host structures, Urbach energy $E_{u}$ has been determined, for doped and undoped samples through the equations:

$$
\begin{gathered}
\operatorname{Ln}(\alpha(h \nu))=\operatorname{Ln}\left(\alpha_{0}\right)+\frac{h \nu}{E_{u}}, \\
E_{u}=\alpha(h \nu)\left(\frac{d[\alpha(h \nu)]}{d[h \nu]}\right)^{-1}=h\left[\frac{d}{d \nu}(\operatorname{Ln} \alpha(\nu))\right]^{-1},
\end{gathered}
$$

where $\alpha(h \nu)$ represents, for each sample, the experimentally deduced optical absorption profile. 


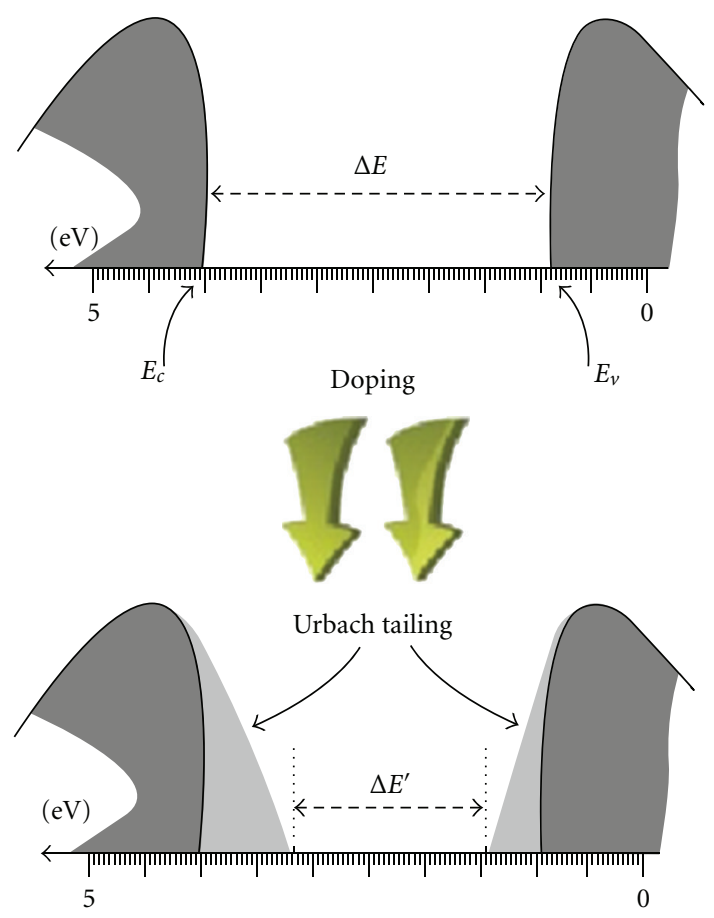

FIGURE 1: Urbach tails occurrence scheme.

TABLE 1: Values of Urbach energy for doped and undoped nanofilms.

\begin{tabular}{lcc}
\hline & Nanofilm & $E_{U}(\mathrm{meV})$ \\
\hline \multirow{2}{*}{ BSO } & Un-doped & 842.4 \\
& Doped & 304.5 \\
\hline \multirow{2}{*}{ BTO } & Un-doped & 564.6 \\
& Doped & 559.2 \\
\hline \multirow{2}{*}{ BGO } & Un-doped & 448.7 \\
& Doped & 430.5 \\
\hline
\end{tabular}

The width of the localized states (band tail energy or Urbach energy $E_{u}$ ) has been estimated (Table 1) from the slopes of the plots of $(\operatorname{Ln} \alpha(\nu))$ versus energy $h \nu$.

\section{Results and Discussion}

As stated earlier, the Lattice Compatibility Theory LCT refers to the behavior of the doping element intrinsic lattice inside the hosting edifice. In the actual situation, the location of holes in both doping agent oxide, BSO, BTO, and BGO lattice structures along with the nature of the highest occupied band, is determinant in conventional orbitals patterns-linked geometry framework. In this context, some relevant observations have been recorded. The first concerns annealing process which led to the creation of an important number of nonparamagnetic new centers. The induced change seems to cause reduction of both oxygen and bismuth. This reduction has been associated with strong changes in the magnitude of the photosensitivity and the dark and hence less stability.
The second is that despite having the same structures as $\mathrm{BSO}$, nano-structured BGO and BTO react differently under doping. In fact, according to the results recorded in Table 1, the important decrease of Urbach energy with doping $(\approx 60 \%)$ reveals an increasing order that occurs in wellcrystallized doped bismuth sillenite BSO contrasts unexpectedly the un-doped ones. This change is absent in the case of doped BTO and BGO. As it was postulated that Urbach energy is a material parameter which indicates the shape of transition from the band tail to the mid gap states (Figure 1), a possible cause to this difference can be attributed to the disposition of the doping agent inside the host matrices (Figure $2(\mathrm{a})$ ). In a first possible scheme, $\mathrm{CuO}_{2}$ copper metallic original fcc lattice is incorporated as such inside BSO host matrix (Figure 2(b)). The second scheme is based on the particularities of $\mathrm{CuO}_{2}$ bonding direction $(\pi, 0) /(0, \pi)$. It is known $\mathrm{CuO}_{2}$ layers are simply stacked in fourfold symmetry, without other atoms in the crystal. Bond lengths comparison has been favorable to possible adoption to two adjacent $\mathrm{O}$ atoms from tetragonal bismuth as edges of $\mathrm{CuO}_{2}$ lattices (Figure 2(c)). Finally, a possible scheme is based on the magnetic properties of the $\mathrm{Cu}_{2} \mathrm{O}_{3}$ spin lattices (Figure 2(d)). In this case, a consolidated stabilization inside the host structure is implemented by symmetrically sharing B-O bands hexagonal bonds. This stability has been recorded by Troth and Dainty [20]. These searchers confirmed in their studies on holographic interferometry and anisotropic self-diffraction in sillenite nano-materials that $\mathrm{Cu}-\mathrm{O}$ bounds in $\mathrm{Cu}_{2} \mathrm{O}_{3}$ edifices are mainly caused by $3 \mathrm{~d}^{9}$ configuration of the doping agent ion in a tetrahedral field [20]. On the other hand, it has been confirmed [21-25] that BGO and BTO present distorted octahedral the bismuth-oxygen coordination made 


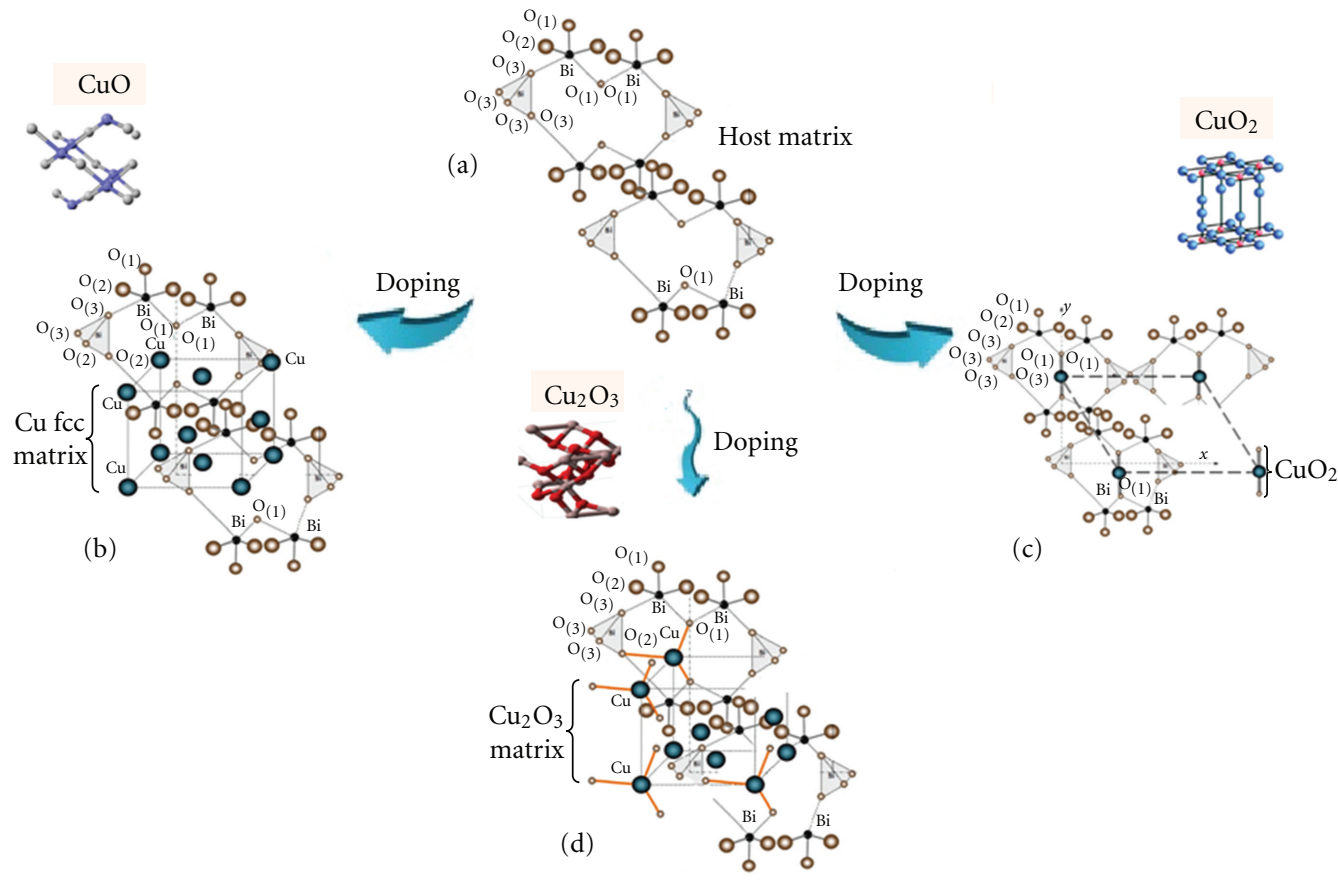

Figure 2: Polyhedra structure in BSO matrix. (a): Un-doped, (b): $\mathrm{CuO}$ doped, (c): $\mathrm{CuO}_{2}$ doped, and (d): $\mathrm{Cu}_{2} \mathrm{O}_{3}$ doped.

up by five oxygen atom and a lone-pair playing the role of the sixth anion. The actually presented Lattice Compatibility Theory (LCT) features are in good agreement with the precedent analyses as well as the notions of lone-pair orbitals, misfit dislocation, and critical layer thickness [26-30]. The disparity and the introduced uncertainty are recorded for $\mathrm{BSO}, \mathrm{BTO}$, and BGO nano-materials plea in favor of this theory as long as it explains the lattice compatibility, the interfacial potential energy, and the strain-associated structural accommodation which were monitored exclusively in the case of BSO.

\section{Conclusion}

In this work we have tried to give a prelude to the Lattice Compatibility Theory (LCT) as an explanative guide for understanding Urbach tailing features inside some nanomaterials. The stability of doping agents in host matrices has been the main fundament to this theory, as it was recorded exclusively in some particular lattices. In order to implement thoroughbred fundaments to this theory, it is necessary to investigate the growth kinetics of differently doped oxides in terms of interaction between host matrix and doping agents' intrinsic structures.

\section{Acknowledgment}

The author wishes to acknowledge the three anonymous reviewers for their detailed and helpful comments to the paper.

\section{References}

[1] N. F. Mott, "Nobel Prize Lecture," 1977.

[2] N. F. Mott, Conduction in Non-Crystalline Materials, Calrendon Press, Oxford, UK, 2nd edition, 1993.

[3] N. F. Mott, Metal-Insulator Transitions, Taylor \& Francis, London, UK, 1974.

[4] F. Urbach, "The long-wavelength edge of photographic sensitivity and of the electronic absorption of solids," Physical Review, vol. 92, no. 5, p. 1324, 1953.

[5] G. Fanchini and A. Tagliaferro, "Disorder and Urbach energy in hydrogenated amorphous carbon: a phenomenological model," Applied Physics Letters, vol. 85, no. 5, pp. 730-732, 2004.

[6] J. D. Dow and D. Redfield, "Toward a unified theory of Urbach's rule and exponential absorption edges," Physical Review B, vol. 5, no. 2, pp. 594-610, 1972.

[7] J. Zhou, Z. Zou, A. K. Ray, and X. S. Zhao, "Preparation and characterization of polycrystalline bismuth titanate $\mathrm{Bi}_{12} \mathrm{TiO}_{20}$ and its photocatalytic properties under visible light irradiation," Industrial and Engineering Chemistry Research, vol. 46, no. 3, pp. 745-749, 2007.

[8] P. Lacovara, "Room-temperature diode-pumped Yb: YAG laser," Optics Letters, no. 16, pp. 1089-1091, 1991.

[9] S. E. Lin, Y. L. Kuo, C. H. Chou, and W. C. J. Wei, "Characterization of electrolyte films deposited by using RF magnetron sputtering a $20 \mathrm{~mol} \%$ gadolinia-doped ceria target," Thin Solid Films, vol. 518, no. 24, pp. 7229-7232, 2010.

[10] S. Tekeli and U. Demir, "Colloidal processing, sintering and static grain growth behaviour of alumina-doped cubic zirconia," Ceramics International, vol. 31, no. 7, pp. 973-980, 2005.

[11] C. H. Weng and W. C. J. Weiw, "Synthesis and properties of homogeneous $\mathrm{Nb}$-doped bismuth oxide," Journal of the American Ceramic Society, vol. 93, no. 10, pp. 3124-3129, 2010. 
[12] S. Lanfredi, J. F. Carvalho, and A. C. Hernandes, "Electric and dielectric properties of $\mathrm{Bi}_{12} \mathrm{TiO}_{20}$ single crystals," Journal of Applied Physics, vol. 88, no. 1, pp. 283-287, 2000.

[13] S. Lanfredi and M. A. L. Nobre, "Conductivity mechanism analysis at high temperature in bismuth titanate: a single crystal with sillenite-type structure," Applied Physics Letters, vol. 86, no. 8, Article ID 081916, 3 pages, 2005.

[14] D. Dirksen and G. Von Bally, "Holographic double-exposure interferometry in near real time with photorefractive crystals," Journal of the Optical Society of America B, vol. 11, no. 9, pp. 1858-1863, 1994.

[15] A. A. Kamshilin and M. P. Petrov, "Continuous reconstruction of holographic interferograms through anisotropic diffraction in photorefractive crystals," Optics Communications, vol. 53, no. 1, pp. 23-26, 1985.

[16] S. Mallick, D. Rouède, and A. G. Apostolidis, "Efficiency and polarization characteristics of photorefractive diffraction in a $\mathrm{Bi}_{12} \mathrm{SiO}_{20}$ crystal," Journal of the Optical Society of America, vol. 4, no. 8, pp. 1247-1259, 1987.

[17] P. Günter and J.-P. Huignard, Photorefractive Materials and Their Applications: Fundamental Phenomena, vol. 61 of Topics in Applied Physics, Springer, Berlin, Germany, 1988.

[18] Y. Dimitriev, K. Krupchanska, Y. Yvanova, and A. Staneva, "Sol-gel synthesis ofi materials in the system $\mathrm{Bi}_{2} \mathrm{O}_{3}-\mathrm{SiO}_{2}$," Journal of the University of Chemical Technology and Metallurgy, vol. 45, no. 1, pp. 39-42, 2010.

[19] Y. A. Borovlev, N. V. Ivannikova, V. N. Shlegel, Y. V. Vasiliev, and V. A. Gusev, "Progress in growth of large sized BGO crystals by the low-thermal-gradient Czochralski technique," Journal of Crystal Growth, vol. 229, no. 1, pp. 305-311, 2001.

[20] R. C. Troth and J. C. Dainty, "Holographic interferometry using anisotropic self-diffraction in $\mathrm{Bi}_{12} \mathrm{SiO}_{20}$, Optics Letters, vol. 16, pp. 53-55, 1991.

[21] C. Elissalde and J. Ravez, "Ferroelectric ceramics: defects and dielectric relaxations," Journal of Materials Chemistry, vol. 11, no. 8, pp. 1957-1967, 2001.

[22] R. J. Betsch and W. B. White, "Vibrational spectra of bismuth oxide and the sillenite-structure bismuth oxide derivatives," Spectrochimica Acta A, vol. 34, no. 5, pp. 505-514, 1978.

[23] M. H. Tuilier, H. Dexpert, P. Lagarde, M. Devalette, and N. Khachani, "An exafs study of the local distortions in some $\mathrm{Bi}_{12}\left[A_{\alpha}^{x+} B_{\beta}^{y+}\right] \mathrm{O}_{20}$ oxides with sillenite-type structure," Journal of Physics and Chemistry of Solids, vol. 48, no. 8, pp. 707-711, 1987.

[24] Y. Nagao and Y. Mimura, "Properties of $\mathrm{Bi}_{12} \mathrm{SiO}_{20}$ single crystals containing first row transition metal," Materials Research Bulletin, vol. 24, no. 2, pp. 239-246, 1989.

[25] M. Ichimura and J. Narayan, "Atomic study of dislocation nucleation in Ge/(001)Si heterostructures," Philosophical Magazine A, vol. 72, p. 281, 1995.

[26] W. D. Nix, "Mechanical properties of thin films," Metallurgical Transactions A, vol. 20, no. 11, pp. 2217-2245, 1989.

[27] I. A. Ovid'ko, "Misfit dislocation walls in solid films," Journal of Physics Condensed Matter, vol. 11, no. 34, pp. 6521-6527, 1999.

[28] L. B. Freund, "The mechanics of dislocations in strained-layer semiconductor materials," in Advances in Applied Mechanics, W. Hutchinson and T. Y. Wu, Eds., pp. 1-66, Academic Press, New York, NY, USA, 1993.

[29] E. A. Fitzgerald, "Dislocations in strained-layer epitaxy: theory, experiment, and applications," Materials Science Reports, vol. 7, no. 3, pp. 87-142, 1991.
[30] J. H. Van der Merwe, "Strain relaxation in epitaxial overlayers," Journal of Electronic Materials, vol. 20, no. 10, pp. 793-803, 1991. 

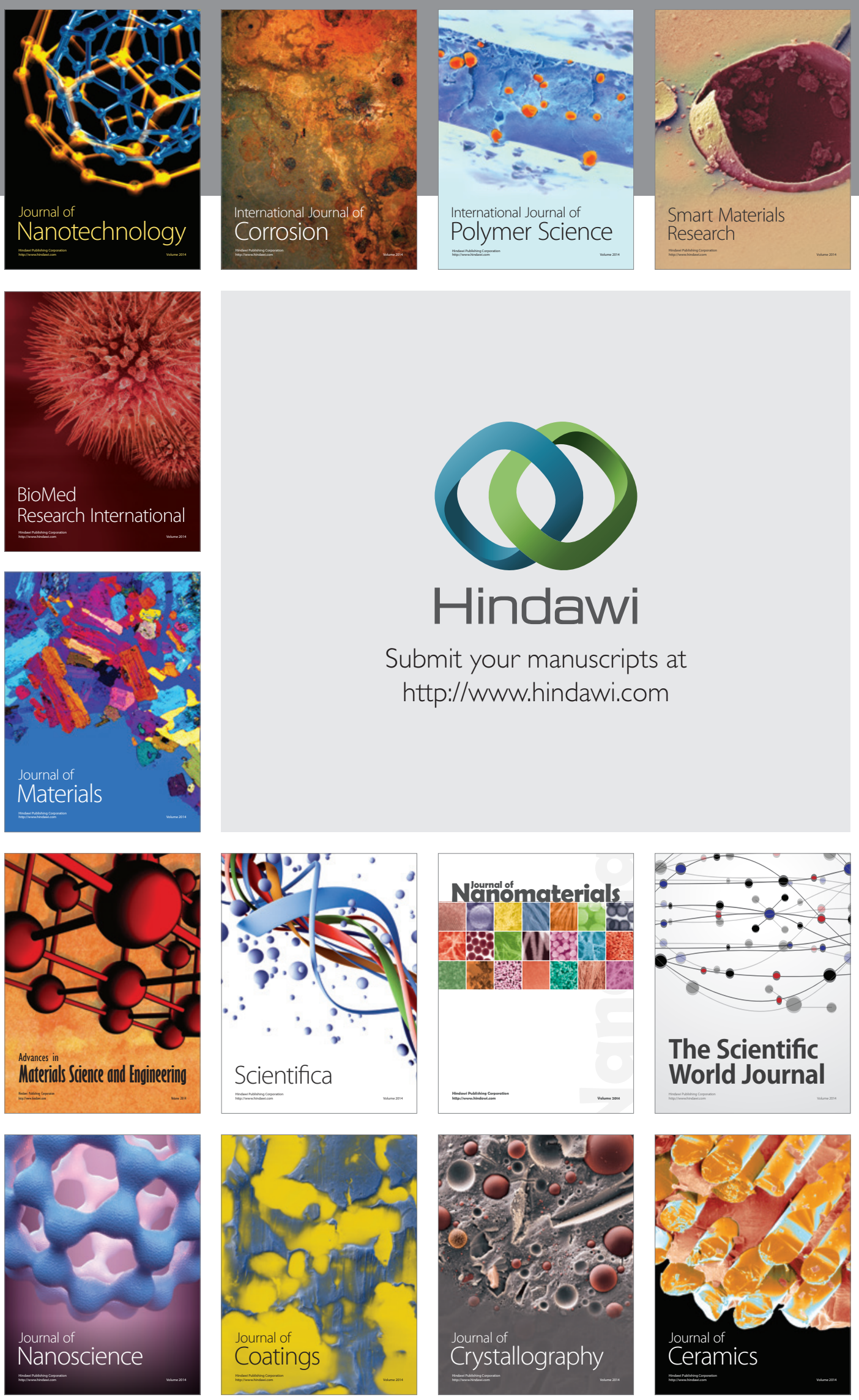

The Scientific World Journal

Submit your manuscripts at

http://www.hindawi.com

\section{World Journal}

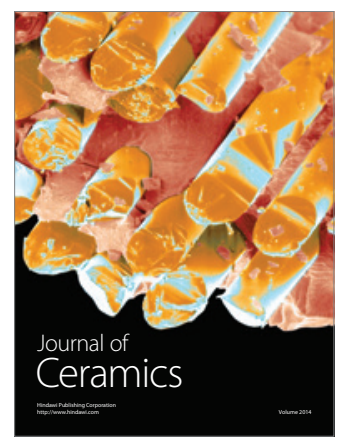

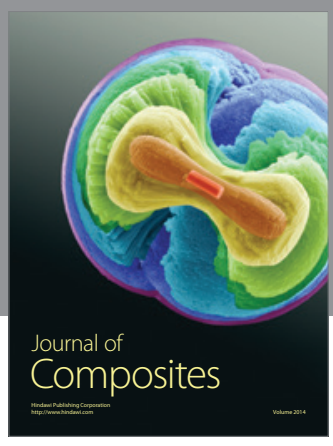
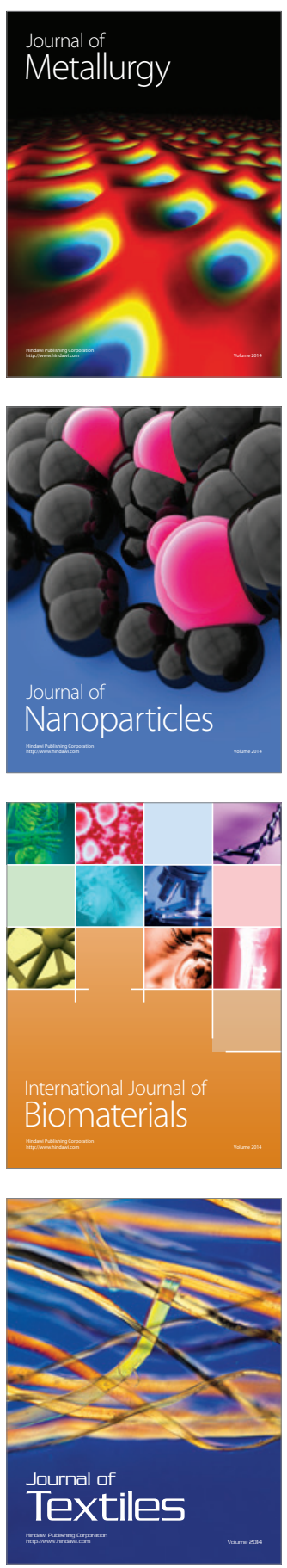